\title{
A STRONG LAW OF LARGE NUMBERS FOR AANA RANDOM VARIABLES IN A HILBERT SPACE AND ITS APPLICATION
}

\author{
Mi-HwA Ko
}

\begin{abstract}
In this paper we introduce the concept of asymptotically almost negatively associated random variables in a Hilbert space and obtain the strong law of large numbers for a strictly stationary asymptotically almost negatively associated sequence of $\mathrm{H}$-valued random variables with zero means and finite second moments. As an application we prove a strong law of large numbers for a linear process generated by asymptotically almost negatively random variables in a Hilbert space with this result.
\end{abstract}

\section{Introduction}

A finite family $\left\{Y_{i}, 1 \leq i \leq n\right\}$ of real-valued random variables is said to be associated if for any coordinatewise increasing functions $f, g$ : $\mathbb{R}^{n} \rightarrow \mathbb{R}$

$$
\operatorname{Cov}\left(f\left(Y_{1}, \cdots, Y_{n}\right), g\left(Y_{1}, \cdots, Y_{n}\right)\right) \geq 0
$$

whenever this covariance exists. A finite family $\left\{Y_{i}, 1 \leq i \leq n\right\}$ is said to be negatively associated(NA) if for any disjoint subsets $A, B \subset$ $\{1, \cdots, n\}$ and any real coordinatewise nondecreasing functions $f$ on $\mathbb{R}^{A}, g$ on $\mathbb{R}^{B}$,

$$
\operatorname{Cov}\left(f\left(Y_{i}, i \in A\right), g\left(Y_{j}, j \in B\right)\right) \leq 0
$$

whenever the covariance exists. An infinite family of random variables is associated(negatively associated) if every finite subfamily is associated(negatively associated). These concepts of dependence were

Received January 12, 2010. Accepted February 22, 2010.

Key words and phrases: Almost sure convergence, linear process in a Hilbert space, asymptotically almost negatively associated, linear operator, Hilbert space valued random variable.

2000 AMS Subject Classifications: 60F05;60F17;60G10. 
introduced by Esary, Proschan and Walkup(1967) and Joag-Dev and Proschan(1983), respectively.

From notion of negative association we can consider the following dependence notion.

A sequence $\left\{Y_{n}, n \geq 1\right\}$ of random variables is called asymptotically almost negatively associated(AANA) if there is a nonnegative sequence $q(m) \rightarrow 0$ such that

$$
\begin{gathered}
\operatorname{Cov}\left(f\left(Y_{m}\right), g\left(Y_{m+1}, \cdots, Y_{m+k}\right)\right) \\
\leq q(m)\left(\operatorname{Var}\left(f\left(Y_{m}\right)\right) \operatorname{Var}\left(g\left(Y_{m+1}, \cdots, Y_{m+k}\right)\right)\right)^{\frac{1}{2}}
\end{gathered}
$$

for all $m, k \geq 1$ and for all coordinatewise increasing continuous functions $f$ and $g$ whenever the right hand side of (1.1) is finite. This definition was introduced by Chandra and Ghosal(1996a, 1996b).

Note that the concept of AANA is weaker than negative association, that is, the family of AANA sequences contains NA(in particular, independent) sequence and some more sequences of random variables which are not much deviated from being negatively associated.

Chandra and Ghosal(1996a) proved the strong law of large numbers of Marcinkiewicz and Zygmund for AANA random variables and they(1996b) also obtained the strong laws of large numbers for weighted averages of AANA random variables. Recently, Kim et al.(2004) obtained the Häjeck - Renyi type inequality for AANA random variables and proved the strong law of large number by applying this inequality.

Let $\left\{\xi_{1}, \cdots, \xi_{m}\right\}$ be a sequence of $\mathbb{R}^{d}$-valued random vectors. $\left\{\xi_{1}, \cdots\right.$, $\left.\xi_{m}\right\}$ is said to be associated if $\operatorname{Cov}\left(f\left(\xi_{1}, \cdots, \xi_{m}\right), g\left(\xi_{1}, \cdots, \xi_{m}\right)\right) \geq 0$ for any nondecreasing functions $f$ and $g$ on $\mathbb{R}^{m d}$, such that the covariance exists. This definition was introduced by Burton, Darbrowski and Dehling(1986).

As Burton, Dabrowski and Dehling(1986) introduced the definition of association for random vectors with values in $\mathbb{R}^{d}$ and random variables with values in a Hilbert space, Ko(2009) and Ko et al.(2009) gave the definition of negative association for random vectors with values in $\mathbb{R}^{d}$ and random variables with values in a Hilbert space as follows. $\left\{\xi_{1}, \cdots, \xi_{m}\right\}$ is said to be negatively associated if $\operatorname{Cov}\left(f\left(\xi_{i}, i \in A\right), g\left(\xi_{j}, j \in B\right)\right) \leq 0$ for any disjoint subsets $A, B \subset\{1, \cdots, n\}$ and for any nondecreasing functions $f$ on $\mathbb{R}^{d|A|}, g$ on $\mathbb{R}^{d|B|}$ such that the covariance exists where $|A|$ is the cardinality of $\mathrm{A}$.

Now by the similar idea we can define the concept of AANA in $\mathbb{R}^{d}$ as follows: Let $\left\{\xi_{n}, n \geq 1\right\}$ be a sequence of $\mathbb{R}^{d}$-valued random vectors. 
$\left\{\xi_{n}, n \geq 1\right\}$ is said to be AANA if there exists a nonnegative sequence $q(m) \rightarrow 0$ such that for all $m, k \geq 1$,

$$
\begin{aligned}
& \operatorname{Cov}\left(f\left(\xi_{m}\right), g\left(\xi_{m+1}, \cdots, \xi_{m+k}\right)\right) \\
& \leq q(m)\left(\operatorname{Var}\left(f\left(\xi_{m}\right)\right) \operatorname{Var}\left(g\left(\xi_{m+1}, \cdots, \xi_{m+k}\right)\right)\right)^{\frac{1}{2}}
\end{aligned}
$$

for every coordinatewise increasing continuous functions $f: \mathbb{R}^{d} \rightarrow \mathbb{R}$ and $g: \mathbb{R}^{k d} \rightarrow \mathbb{R}$ so that the right-hand side of (1.2) is finite.

We also extend the concept of AANA for random vectors with values in $\mathbb{R}^{d}$ to random vectors with values in a separable Hilbert space as follows : Let $\left\{\xi_{n}, n \geq 1\right\}$ be a sequence of random variables taking values in a Hilbert space $(H .<\cdot, \cdot\rangle) .\left\{\xi_{n}, n \geq 1\right\}$ is said to be AANA if for some orthonormal basis $\left\{e_{j}, j \geq 1\right\}$ of $\mathrm{H}$ and for any $d \geq 1$ the $d$-dimensional sequence $\left(<\xi_{i}, e_{1}>, \cdots,<\xi_{i}, e_{d}>\right), i \geq 1$ is AANA.

Let $\mathrm{H}$ be a separable real Hilbert space with the norm $\|\cdot\|_{H}$ generated by an inner product, $\langle\cdot, \cdot\rangle_{H}$ and let $\left\{e_{k}, k \geq 1\right\}$ be an orthonormal basis in $\mathrm{H}$. Let $L(H)$ be the class of bounded linear operators from $\mathrm{H}$ to $\mathrm{H}$ and denote by $\|\cdot\|_{L(H)}$ its usual norm. Let $\left\{\xi_{k}, k \in \mathbb{Z}\right\}$ be a strictly stationary sequence of $\mathrm{H}$-valued random variables and $\left\{a_{k}, k \in \mathbb{Z}\right\}$ be a sequence of bounded operator, that is $a_{k} \in L(H)$. We define the stationary linear process in a Hilbert space by

$$
X_{k}=\sum_{j=0}^{\infty} a_{j} \xi_{k-j}, k \in \mathbb{Z} .
$$

(See Melèvède et al.(1997), and Bosq(2003) for more details.) The sequence $\left\{X_{k}, k \in \mathbb{Z}\right\}$ is a natural extension of the multivariate linear processes(Brockwell and Davis(1987), Chap. 11). We define

$$
S_{n}=\sum_{k=1}^{n} X_{k} .
$$

Notice that if $\sum_{j=0}^{\infty}\left\|a_{j}\right\|_{L(H)}<\infty$ and $\left\{\xi_{k}, k \in \mathbb{Z}\right\}$ is a sequence of $\mathrm{H}$ valued i.i.d. random variables centered in $L_{2}(H)$, then it is well known that the series in (1.3) converges almost surely(Araujo and Gine(1980), Chap. 3.2).

In Section 2 we will study the strong law of large numbers for AANA random variables in a Hilbert space and in Section 3 we show the strong law of large numbers for a strictly stationary linear process generated by AANA random variables in a Hilbert space as its application. 


\section{Results}

Lemma 2.1(Chandra, Ghosal (1996 a,b)) Let $\left\{Y_{n}, n \geq 1\right\}$ be a sequence of mean zero, square integrable random variables such that (1.1) holds for $1 \leq m<k+m \leq n$ and for all coordinatewise increasing continuous functions $f$ and $g$ whenever the right-hand side of (1.1) is finite. Let $A^{2}=\sum_{m=1}^{n-1} q^{2}(m)$ and $\sigma_{k}^{2}=E Y_{k}^{2}, k \geq 1$. Then

$$
E\left(\max _{1 \leq j \leq n} \sum_{i=1}^{j} Y_{i}\right)^{2} \leq 2\left(A+\left(1+A^{2}\right)^{\frac{1}{2}}\right)^{2} \sum_{k=1}^{n} \sigma_{k}^{2},
$$

From Lemma 2.1 we obtain the following maximal inequality for $\mathrm{H}$ valued AANA random variables:

Lemma 2.2 Let $\left\{\xi_{i}, 1 \leq i \leq n\right\}$ be a sequence of mean zero, square integrable $\mathrm{H}$-valued random variables such that (1.2) holds for $1 \leq m \leq k+$ $m \leq n$ and for all coordinatewise increasing continuous functions $f$ and $g$ whenever the right-hand side of (1.2) is finite.Let $A^{2}=\sum_{m=1}^{n-1} q^{2}(m)$. Then

$$
P\left\{\max _{1 \leq j \leq n}\left\|\sum_{i=1}^{j} \xi_{i}\right\| \geq \epsilon\right\} \leq 2 \epsilon^{-2}\left(A+\left(1+A^{2}\right)^{\frac{1}{2}}\right)^{2} \sum_{i=1}^{n} E\left\|\xi_{i}\right\|^{2} .
$$

Proof It follows from Lemma 2.1 that

$$
\begin{aligned}
\text { (2.3) } E \max _{1 \leq j \leq n}\left\|\sum_{i=1}^{j} \xi_{i}\right\|^{2} & =E \max _{1 \leq j \leq n} \sum_{k=1}^{\infty}\left(\sum_{i=1}^{j}<\xi_{i}, e_{k}>\right)^{2} \\
& \leq \sum_{k=1}^{\infty} E\left(\max _{1 \leq j \leq n} \sum_{i=1}^{j}<\xi_{i}, e_{k}>\right)^{2} \\
& \leq 2 \sum_{k=1}^{\infty}\left(A+\left(1+A^{2}\right)^{\frac{1}{2}}\right)^{2} \sum_{1=1}^{n} E\left(<\xi_{i}, e_{k}>\right)^{2} \\
& \leq 2\left(A+\left(1+A^{2}\right)^{\frac{1}{2}}\right)^{2} \sum_{i=1}^{n} E \sum_{k=1}^{\infty}\left(<\xi_{i}, e_{k}>\right)^{2} \\
& =2\left(A+\left(1+A^{2}\right)^{\frac{1}{2}}\right)^{2} \sum_{i=1}^{n} E\left\|\xi_{i}\right\|^{2}
\end{aligned}
$$

which yields (2.2). 
Theorem 2.3 Let $\left\{\xi_{n}, n \geq 1\right\}$ be an AANA sequence of H-valued random variables with $E \xi_{n}=0$ and $E\left\|\xi_{n}\right\|^{2}<\infty$. Assume

$$
\sum_{i=1}^{\infty} E\left\|\xi_{i}\right\|^{2}<\infty .
$$

Then $S_{n}$ converges almost surely, where $S_{n}=\xi_{1}+\cdots+\xi_{n}$.

Proof Let $\epsilon>0$. Then

$$
\begin{aligned}
P\left\{\sup _{k, m \geq n}\left\|S_{k}-S_{m}\right\|>\epsilon\right\} \leq & P\left\{\sup _{k \geq n}\left\|S_{k}-S_{n}\right\|>\frac{1}{2} \epsilon\right\} \\
& +P\left\{\sup _{m \geq n}\left\|S_{m}-S_{n}\right\|>\frac{1}{2} \epsilon\right\} \\
\leq & 2 \lim _{N \rightarrow \infty} P\left\{\max _{n \leq k \leq N}\left\|S_{k}-S_{n}\right\|>\frac{1}{2} \epsilon\right\} .
\end{aligned}
$$

Hence, by Lemma 2.2 and (2.4) we have

$$
\begin{aligned}
& P\left\{\sup _{k, m \geq n}\left\|S_{k}-S_{m}\right\|>\epsilon\right\} \leq 8\left(A+\left(1+A^{2}\right)^{\frac{1}{2}}\right)^{2} \epsilon^{-2} \sum_{i=n}^{\infty} E\left\|\xi_{i}\right\|^{2} \rightarrow 0, \\
& \text { as } n \rightarrow \infty \text {, }
\end{aligned}
$$

where A is defined in Lemma 2.2.

Thus we may conclude that the sequence $\left\{S_{n}, n \geq 1\right\}$ is Cauchy almost surely and therefore convergent almost surely.

The above theorem and the Kronecker lemma(cf Stout(1995)) imply the following result.

Theorem 2.4 Let $\left\{\xi_{n}, n \geq 1\right\}$ be an AANA sequence of H-valued random variables with $E \xi_{n}=0$ and $E\left\|\xi_{n}\right\|^{2}<\infty, n \geq 1$. Assume

$$
\sum_{j=1}^{\infty} j^{-2} E\left\|\xi_{j}\right\|^{2}<\infty .
$$

Then, as $n \rightarrow \infty$ we have $n^{-1} S_{n} \rightarrow 0$ almost surely, where $S_{n}=\xi_{1}+$ $\cdots+\xi_{n}$.

Corollary 2.5 Let $\left\{\xi_{n}, n \geq 1\right\}$ be an AANA sequence of $R^{d \text {-valued }}$ random vectors, centered at expectations and finite second moments. If (2.4) holds, then $S_{n}$ converges almost surely. 
Corollary 2.6 Let $\left\{\xi_{n}, n \geq 1\right\}$ be an AANA sequence of $R^{d}$-valued random vectors, centered at expectations and finite second moments. If (2.5) holds, then, as $n \rightarrow \infty, n^{-1} S_{n} \rightarrow 0$ a.s..

\section{Applications}

Now we apply the strong law of large numbers for AANA random variables in a Hilbert space to the linear processes.

Lemma 3.1 Let $\left\{a_{k}, k \in \mathbb{Z}\right\}$ be a sequence of bounded linear operators and let $\widetilde{a_{i}}=\sum_{j=i+1}^{\infty} a_{j}$. If $\sum_{j=0}^{\infty} j\left\|a_{j}\right\|<\infty$ then $\sum_{j=0}^{\infty}\left\|\widetilde{a_{j}}\right\|<\infty$.

Proof. Clearly we have $\sum_{j=0}^{\infty}\left\|\widetilde{a_{j}}\right\| \leq \sum_{j=0}^{\infty} \sum_{i=j+1}^{\infty}\left\|a_{i}\right\|=\sum_{j=0}^{\infty} j\left\|a_{j}\right\|$ by induction.

Theorem 3.2 Let $\left\{\xi_{k}, k \in \mathbb{Z}\right\}$ be a strictly stationary asymptotically almost negatively associated sequence of $\mathrm{H}$-valued random variables with $E \xi_{n}=0$ and $E\left\|\xi_{n}\right\|^{2}<\infty$. Let $\left\{a_{k}, k \in \mathbb{Z}\right\}$ be a sequence of bounded linear operators on $\mathrm{H}$ satisfying

$$
\sum_{j=0}^{\infty} j\left\|a_{j}\right\|_{L(H)}<\infty
$$

Define the stationary Hilbert space process by $X_{k}=\sum_{j=0}^{\infty} a_{j} \xi_{k-j}, k \in$ $\mathbb{Z}$. Assume $\sum_{j=1}^{\infty} j^{-2} E\left\|\xi_{j}\right\|^{2}<\infty$. Then

$$
n^{-1} \sum_{k=1}^{n} X_{k} \rightarrow 0 \text { a.s. }
$$


Proof Letting $\widetilde{a_{j}}=\sum_{i=j+1}^{\infty} a_{i}$ and $Y_{k}=\sum_{j=0}^{\infty} \widetilde{a_{j}} \xi_{k-j}$, which is well define since $\sum_{j=0}^{\infty}\left\|\widetilde{a_{j}}\right\|_{L(H)}<\infty$ according to Lemma 3.1, we have

$$
\begin{aligned}
X_{k} & =\sum_{j=0}^{\infty} a_{j} \xi_{k-j} \\
& =a_{0} \xi_{k}+\sum_{j=1}^{\infty} a_{j} \xi_{k-j} \\
& =\left(\sum_{j=0}^{\infty} a_{j}\right) \xi_{k}-\widetilde{a_{0}} \xi_{k}-\sum_{j=1}^{\infty}\left(\widetilde{a_{j}}-\widetilde{a}_{j-1}\right) \xi_{k-j} \\
& =\left(\sum_{j=0}^{\infty} a_{j}\right) \xi_{k}+Y_{k-1}-Y_{k},
\end{aligned}
$$

which implies that

$$
\sum_{k=1}^{n} X_{k}=\left(\sum_{j=0}^{\infty} a_{j}\right) \sum_{k=1}^{n} \xi_{k}+Y_{0}-Y_{n} .
$$

Using Theorem 2.4 on $\left(\sum_{j=0}^{\infty} a_{j}\right) \sum_{k=1}^{n} \xi_{k}$, the theorem is proved if

$$
n^{-1} Y_{0} \rightarrow 0 \text { a.s. and } n^{-1} Y_{n} \rightarrow 0 \text { a.s., as } n \rightarrow \infty \text {. }
$$

To prove (3.3) we note that

$$
E\left\|Y_{k}\right\| \leq \sum_{j=0}^{\infty}\left\|\widetilde{a_{j}}\right\| E\left\|\xi_{k-j}\right\|=E\left\|\xi_{k-j}\right\| \sum_{j=0}^{\infty}\left\|\widetilde{a_{j}}\right\|_{L(H)}<\infty
$$

and that $\sum_{n=1}^{\infty} P(\|X\|>n)<\infty \Leftrightarrow E\|X\|<\infty$.

Hence

$$
\sum_{n=1}^{\infty} P\left\{n^{-1}\left\|Y_{n}\right\|>\epsilon\right\}=\sum_{n=1}^{\infty} P\left\{n^{-1}\left\|Y_{0}\right\|>\epsilon\right\}<\infty
$$

for any $\epsilon>0$, which yields (3.3).

Corollary 3.3 Let $\left\{X_{k}, k \in \mathbb{Z}\right\}$ be a $d$-dimensional linear process of the form $X_{k}=\sum_{j=0}^{\infty} a_{j} \xi_{k-j}$ where $\left\{\xi_{k}, k \in \mathbb{Z}\right\}$ is a strictly stationary sequence of $d$-dimensional asymptotically almost negatively associated random vectors with $E \xi_{n}=0, E\left\|\xi_{n}\right\|<\infty$ and $E\left\|\xi_{n}\right\|^{2}<\infty$ and $\left\{a_{k}, k \in \mathbb{Z}\right\}$ is a sequence of $d \times d$ matrix with $\sum_{j=0}^{\infty} j\left\|a_{j}\right\|<\infty$. 
Assume

$$
\sum_{j=1}^{\infty} j^{-2} E\left\|\xi_{j}\right\|^{2}<\infty
$$

Then, as $n \rightarrow \infty$

$$
n^{-1} \sum_{k=1}^{n} X_{k} \rightarrow 0 \text { a.s. }
$$

Theorem 3.4 Let $\left\{\xi_{k}, k \in \mathbb{Z}\right\}$ be a strictly stationary sequence of $\mathrm{H}$-valued asymptotically almost negatively associated random variables with $E \xi_{0}=0, E\left\|\xi_{0}\right\|<\infty$ and let $\left\{a_{k}, k \in \mathbb{Z}\right\}$ be a sequence of bounded linear operators on $\mathrm{H}$ satisfying (3.1). Then

$$
n^{-1} \sum_{k=1}^{n} \xi_{k} \rightarrow 0 \text { a.s. implies } n^{-1} \sum_{k=1}^{n} X_{k} \rightarrow 0 \text { a.s. }
$$

Proof The proof of Theorem 3.3 is similar to that of Theorem 3.2.

Corollary 3.5 Let $\left\{X_{k}, k \in \mathbb{Z}\right\}$ be a $d$-dimensional linear process of the form $X_{k}=\sum_{j=0}^{\infty} a_{j} \xi_{k-j}$, where $\left\{\xi_{k}, k \in \mathbb{Z}\right\}$ is a strictly stationary sequence of $d$-dimensional asymptotically almost negatively associated random vectors with $E \xi_{0}=0, E\left\|\xi_{0}\right\|<\infty$ and $\left\{a_{k}, k \in \mathbb{Z}\right\}$ is a sequence of $d \times d$ matrix with $\sum_{j=0}^{\infty} j\left\|a_{j}\right\|<\infty$. Then

$$
n^{-1} \sum_{k=1}^{n} \xi_{k} \rightarrow 0 \text { a.s. implies } n^{-1} \sum_{k=1}^{n} X_{k} \rightarrow 0 \text { a.s. }
$$

Acknowledgement The author would like to thank the referees for the careful reading of the manuscript and useful suggestions. This work was supported by the Korean Research Foundation Grant funded by the Korean government(MOEHRD(KRF-2006-353-C00006)). 


\title{
References
}

[1] Araujo, A. and Gine, E.(1980) The Central Limit Theorem for Real and Banach Valued Random Variables, John Wiley and Sons.

[2] Bosq, D. (2004) Berry-Essen inequality for linear processes in Hilbert spaces, Statist. Probab. Lett. 63 243-247

[3] Brockwell, P. and Davis, R.(1987) Time series, Theory and Method. Springer, Berlin

[4] Burton, R., Dabrowski, A.R. and Dehling, H.(1986) An invariance principle for weakly associated random vectors, Stochastic Processes Appl. 23 301-306

[5] Chandra, T. K., Ghosal, S. (1996 a). Extensions of the strong law of large numbers of Marcinkiewicz and Zygmund for dependent variables, Acta. Math. Hungar. 32 327-336.

[6] Chandra, T. K., Ghosal, S. (1996 b). The strong law of large numbers for weighted averages under dependence assumptions, J. Theor. Probab. 9 797-809.

[7] Esary, J. Proschan, F. and Walkup, D. (1967). Association of random variables with applications, Ann. Math. Stat. 38 1466-1474.

[8] Joag-Dev, K. and Proschan, F. (1983) Negative association of random variables with applications, Ann. Statist. 11 286-295

[9] Kim, T.S., Ko, M.H. and Lee I.H. (2004). On the strong law for asymptotically almost negatively associated random variables, Rocky Mountain J. Math. 34 979989.

[10] Ko, M.H., Kim, T.S. and Han, K.H. (2009) A note on the almost sure convergence for dependent random variables in a Hilbert space, J. Theor. Probab. 22 506-513

[11] Ko, M.H. (2009) A central limit theorem for linear process in a Hilbert space under negative association, Korean Commun. Stat. 16 687-696

[12] Melèvède, F., Peligrad, M. and Utev, S. (1997) Sharp conditions for the CLT of linear processes in a Hilbert space, J. Theor. Probab. 10 681-693

[13] Stout, W.F. (1995) Almost sure convergence, Academic, New York.

\author{
Mi-Hwa Ko \\ Department of Statistics, \\ WonKwang University, \\ Jeonbuk 570-749, Korea \\ E-mail: songhack@wonkwang.ac.kr
}

